\title{
Lithography of diamond-like-carbon (DLC) films for use as masters in soft-lithography
}

\author{
Gregory S. Watson ${ }^{\mathrm{a}}$, Sverre Myhra ${ }^{\mathrm{b}}$, Jolanta A. Watson ${ }^{\mathrm{a}}$ \\ ${ }^{a}$ Nanoscale Science and Technology Centre, School of Science, Griffith University; Nathan, \\ Queensland 4111, Australia \\ ${ }^{\mathrm{b}}$ Oxford University Begbroke Science Park, Yarnton, OX5 1PF, UK
}

\begin{abstract}
Micron sized structures/components are commonly employed in a variety of devices (e.g., biosensors, array devices). At present such devices are based on macroscopic technologies. Future applications of differentiated structures/surfaces are expected to place considerable demands on down-sizing technologies, i.e. enable meso/nanoscopic manipulation. An emerging set of methods known collectively as soft lithography is now being utilised for a large variety of applications including micromolding, microfluidic networks and microcontact printing. In particular stamps and elastomeric elements can be formed by transfer of a pattern to a polymer by a master. The 'master' can be fabricated by a variety of techniques capable of producing well-defined surface topographies. Established lithographic techniques used in the microelectronic industry, such as photolithography, are generally used to fabricate such master templates at the micron scale.
\end{abstract}

A number of polymers can be used to transfer patterns. One of the most widely used polymers for pattern transfer has been polydimethylsiloxane (PDMS). The elastomer is chemically resistant, has a low surface energy and readily conforms to different surface topographies. Obtaining a master is the limiting factor in the production of PDMS replicas.

In this study we demonstrate the use of Diamond-Like-Carbon (DLC) as a master template for producing PDMS micro/nano stamps and 3 dimensional PDMS structures. Intricate surface relief patterns were formed on the DLC surface from lithographic techniques by Atomic Force Microscopy (AFM) operated in the electrical conductivity mode.

Attributes of the technique include

- $\quad$ Features with line widths less than $20 \mathrm{~nm}$ can be formed on the DLC.

- The radius of curvature at edges can be less than $10 \mathrm{~nm}$.

- The slope of the features is limited by the aspect ratio of the tip.

- Highly complex shapes can be fashioned.

- $\quad$ Feature depth can be controlled by DLC film thickness and/or by the bias voltage applied.

- The master is highly durable.

- The master relief after patterning is extremely flat.

Keywords: Atomic force microscopy, PDMS, stamps, DLC, Diamond-like-Carbon.

\section{INTRODUCTION}

The invention of the scanning tunnelling microscope (STM), and the subsequent development of a suite of related local probes, known collectively as scanning probe microscopies (SPM), have given the scientific community new tools with which to visualize on the nano- and meso-scale the structure and properties of surfaces and interfaces. Recently it has become apparent that the ability to manipulate purposefully those structures and properties may be an even more important attribute of the SPM family.

A number of potentially enabling SPM-based nano-technologies for manipulation of surfaces have been proposed in recent years. Those are described variously as nano-writing or nano-lithography, probe-based data storage, surface patterning, templating, or nano-machining. The common element is exploitation of the localized interaction between a 
sharp tip and a surface for the purpose of spatially-resolved manipulation of surface structures and/or properties. For instance, oxide structures with a line-width resolution of less than $10 \mathrm{~nm}$ can be written by an SPM probe to a silicon surface or certain metallic substrates by anodic oxidation, e.g. [1-3]; mechanical inscription on a surface can be carried out with a diamond-tipped stiff lever ${ }^{4}$; polymer surfaces can readily be indented, either by tip-induced 'ploughing' or by a thermo-mechanical mechanism ${ }^{5}$; the local magnetic ${ }^{6}$ and ferroelectric structure ${ }^{7}$ can be manipulated by magnetic force and 'electrostatic' force microscopy, respectively. Of greater relevance to the present project is a description of oxidative nano-lithographic patterning of amorphous graphitic carbon films ${ }^{8-10}$. In another AFM-based study the wear characteristics during application of a bias voltage have been investigated for amorphous carbon films ${ }^{11}$.

An emerging set of methods known collectively as soft lithography is now being utilised for a large variety of applications including micromolding, microfluidic networks and microcontact printing [e.g., 12]. The fabrication and use of micro/nano stamps and fluidic channels using PDMS material has been demonstrated in a number of studies [e.g., 1316]. In particular stamps and elastomeric elements can be formed by exposure of a polymer to a template. Established lithographic techniques used in the microelectronic industry, such as photolithography, are generally used to fabricate such master templates at the micron scale.

In this study we demonstrate the use of Diamond-Like-Carbon (DLC) as a template for producing polymer micro/nano stamps and 3-D polymer structures. Intricate surface relief patterns can be formed on the DLC surface from lithographic techniques by Atomic Force Microscopy (AFM) operated in the electrical conductivity mode.

A number of polymers can be used to transfer patterns. One of the most widely used polymers for pattern transfer has been polydimethylsiloxane (PDMS). The elastomer is chemically resistant, has a low surface energy and readily conforms to different surface topographies. Obtaining a master is the limiting factor in the production of PDMS replicas.

\section{EXPERIMENTAL DETAILS}

\subsection{Specimen materials}

Electrically conductive IB-DLC films were deposited onto polished n-type Si substrates. The process technology was an ion beam assisted variation of the generic chemical vapour deposition route. The substrate was maintained at $80^{\circ} \mathrm{C}$ at an ambient pressure of $10^{-6}$ Torr, while being exposed to a partial pressure of polyphenyl ether from a source held at $150^{\circ} \mathrm{C}$. The outcome was a wear-resistant diamond-like carbon film, although with considerable graphitic character, and exhibiting low friction (0.1-0.2 for contact loads up to $100 \mathrm{~N}$ ). RMS surface roughness of the films was in the range 0.25-0.3 nm inferred from AFM imaging over fields of view of $1 \times 1$ and $2 \times 2 \mu \mathrm{m}^{2}$.

The $\mathrm{Si}+$ oxide was an n-type industry-standard wafer (grown by the $\mathrm{Cz}$-method with P-doping leading to a resistivity of 9-15 $\Omega \mathrm{cm}$ ). The substrates had a typical rms roughness of $0.1-0.2 \mathrm{~nm}$, as determined by AFM analysis. A fully dense and uniform oxide layer, of similar rms roughness, was then grown thermally on the substrates. The average thickness of the oxide of $2.4 \mathrm{~nm}$ was determined by ellipsometry. Specimens were cleaned ultrasonically in iso-propyl-alcohol, then rinsed with distilled water and allowed to dry in a clean laminar flow containment.

PDMS (Sylgard ${ }^{\circledR}$-184) was supplied by Dow Corning as a two part silicone elastomer. The base and curing agent were mixed at a 10:1 weight ratio, deposited onto the DLC templates and cured for 1 hour at a temperature of $100^{\circ} \mathrm{C}$ prior to peeling (removal of the polymer from the DLC template) and any subsequent analysis.

\subsection{SPM instrumentation}

The analysis was carried out with JEOL JSTM-4200D and JSPM-4200 multi-technique instruments. The JSPM-4200 was utilised for the subsequent PDMS analysis. A typical sequence of DLC surface modification and analysis proceeded as follows: A large field of view, typically $1-4 \mu^{2}$ was imaged in the contact mode with a lever-imposed force loading of 10 $100 \mathrm{nN}$ and zero bias, in order to ascertain that the chosen surface region was free of any artefacts. The surface modification at a point, or over a small field of view, was then carried out, at particular values of bias voltage, scan speed and force loading. A larger field of view, centred on the location of the surface modification, was then rescanned at zero bias in order to reveal the topographical effects induced by the tip-to-substrate bias. The force-loading was maintained constant throughout the runs, whenever appropriate, as were the parameters used for topographical analysis, in order to improve reproducibility of outcomes. 


\subsection{Probes}

The probe consists of a lever with an integral conical tip attached at its free end. The tip-to-surface point of contact defines the interaction volume, whereby a large amount of information such as topography, strength of in-plane and outof-plane forces is extracted. The point of contact is also that through which purposeful manipulation is effected.

Beam-shaped levers were used in order to ensure that only simple bending modes needed to be considered. The data for the length, width and thickness of the lever were $350 \mu \mathrm{m}, 35 \mu \mathrm{m}$ and $2 \mu \mathrm{m}$, respectively. The radius of curvature at the tip apex, $R_{\text {Tip }}$, aspect ratio (opening half angle) of the tip, $A_{r}$, and surface chemistry $\left(<10 \mathrm{~nm},<10^{\circ}\right.$ and native Si-oxide film or Au-terminated, respectively) were supplied by the suppliers' specifications.

The tips utilised for DLC manipulation were also characterized by 'reverse' imaging over a grid of spikey features; the radii of curvature were consistent with those claimed by the manufacturer. As well, the probes were subjected to I-V characterization before and during experimental runs by investigation of point-contact resistance on a Si-substrate covered by a gold film. Those probes that exhibited contact resistance greater than $10^{10} \Omega$ were discarded.

Many polymers can be considered as 'soft' objects; therefore the imposition of forces at the point of contact will cause deformation and indentation, and will lead to an increase in contact area. Thus it is necessary to use levers with force constants, $k_{N}, \leq 0.1 \mathrm{nNnm}^{-1}$ in order to improve resolution and avoid unintended surface modification. Levers with force constants $\geq 4 \mathrm{nNnm}^{-1}$ result in considerable surface manipulation.

\section{RESULTS AND DISCUSSION}

\subsection{Oxidation in air}

Nano-manipulation by tip-induced local prompt oxidation of electrically conductive DLC material can be carried out to produce well-defined 3-dimensional lithographic patterns ${ }^{17},{ }^{18}$. The extent of spatial resolution for 3-dimensional structures is illustrated by the image and contour line in figure 1. A square pit shown in figure 1 (a) can be obtained by scanning at a sample bias of $4.5 \mathrm{~V}$ for a total duration of $90 \mathrm{~s}(1.5 \mathrm{~ms}$ per pixel over a field of view made up of $256 \times 256$ pixels). The pit is $100 \times 100 \mathrm{~nm}^{2}$ with a depth of $12-13 \mathrm{~nm}$. The corresponding line profile is shown in figure 1 (b). The slope and shape of the side-wall was consistent with the tip apex being described by a parabolic section, and the bottom of the pit was flat to within $\pm 1 \mathrm{~nm}$. It is noteworthy that the 'roughness' of a line drawn along an edge of the pit was less than $5 \mathrm{~nm}$. The in-plane curvature at a corner, and the out-of plane curvature at an edge were both ca. $5 \mathrm{~nm}$.
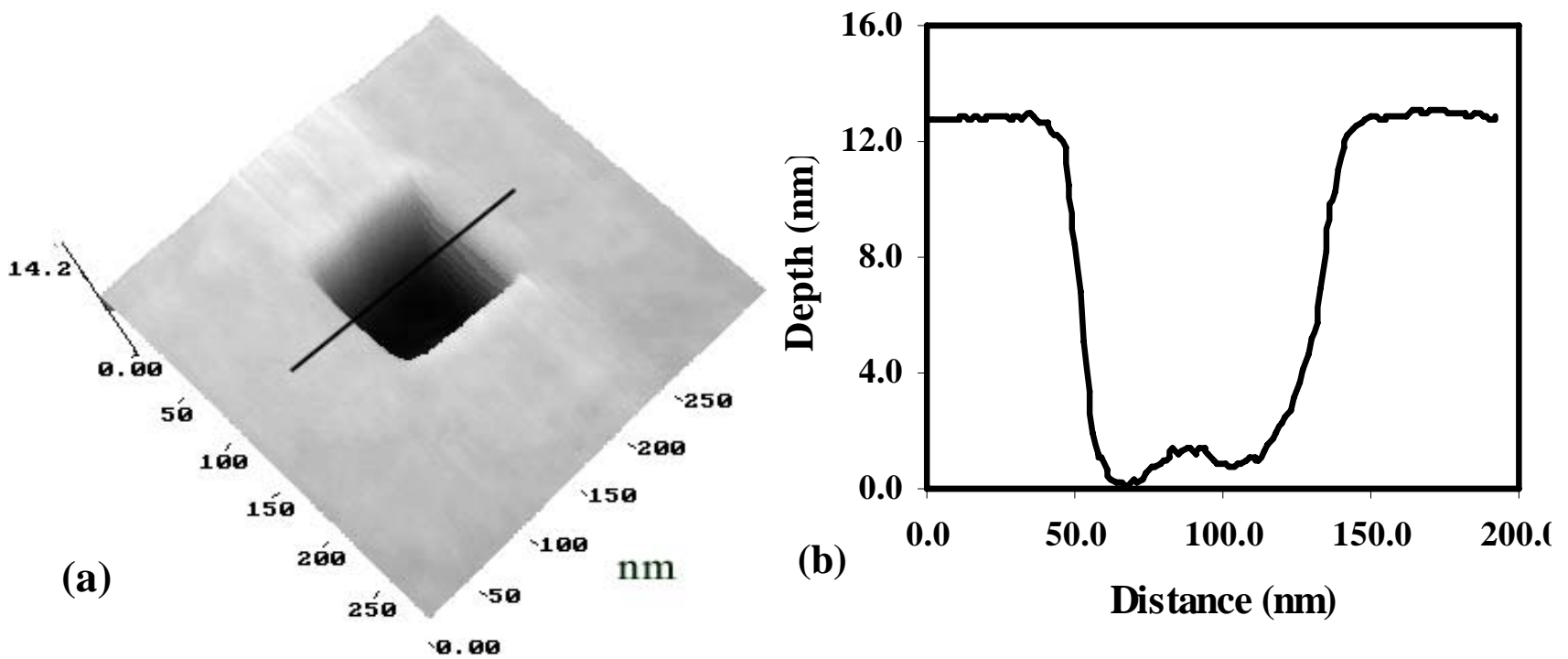

Figure 1 - Contact mode image as a 3 dimensional representation (a) and cross-sectional contour line (b) illustrating the outcome of carrying out tip-induced oxidation in air. A positive sample bias of $4.5 \mathrm{~V}$ was applied for $90 \mathrm{~s}$, with the tip being scanned over a $100 \times 100 \mathrm{~nm}^{2}$ field of view composed of $256 \times 256$ pixels. 
A series of trenches, $500 \mathrm{~nm}$ in length and offset by $100 \mathrm{~nm}$, were each inscribed by a single linear raster of the tip (10 ms per pixel for a total of 128 pixels per line). A 3-dimensional image and a transverse contour line are shown in figure 2 (a) and (b), respectively. The dependence on tip-to-sample bias was explored over the range 5.0-6.5 V. The full width at half-maximum depth was in the range $20-40 \mathrm{~nm}$. The scan direction was from top-left to bottom-right. The application of bias was synchronized with the start of the line scan. Accordingly the irregularities at the beginning of the line are indicative of the time delay for establishing stable (thermal) conditions.

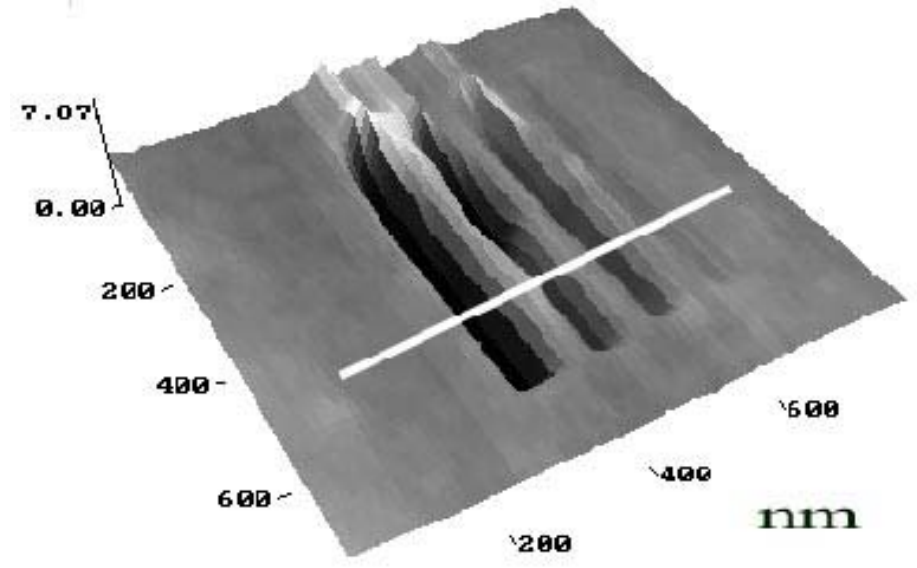

(a)

Q.00

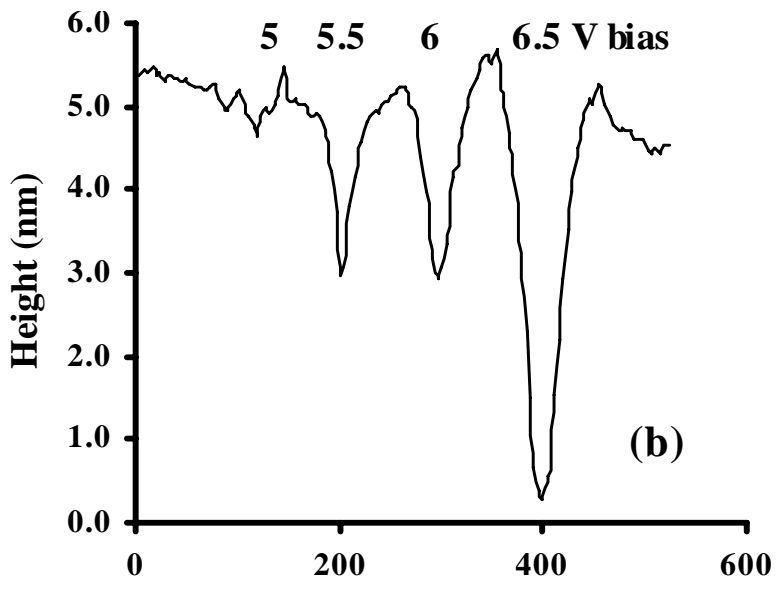

Distance (nm)

Figure 2 - Contact mode image as a 3 dimensional representation (a) and contour lines of trenches (b) inscribed at bias voltages from 5 to $6.5 \mathrm{~V}$. Each line was generated by 128 pixels and a dwell-time of $10 \mathrm{~ms} / \mathrm{pixel}$.

Figure 3 provides an additional illustration of the dependence on deposited energy per unit area. The image shows the outcome of applying bias voltages of $7.5,8.5$ and $9.5 \mathrm{~V}$ to a stationary tip (top to bottom in the grey-scale image). The dwell time of $15 \mathrm{~s}$ per location was constant, as were all other parameters. The contour line through the centres of the pits is shown.
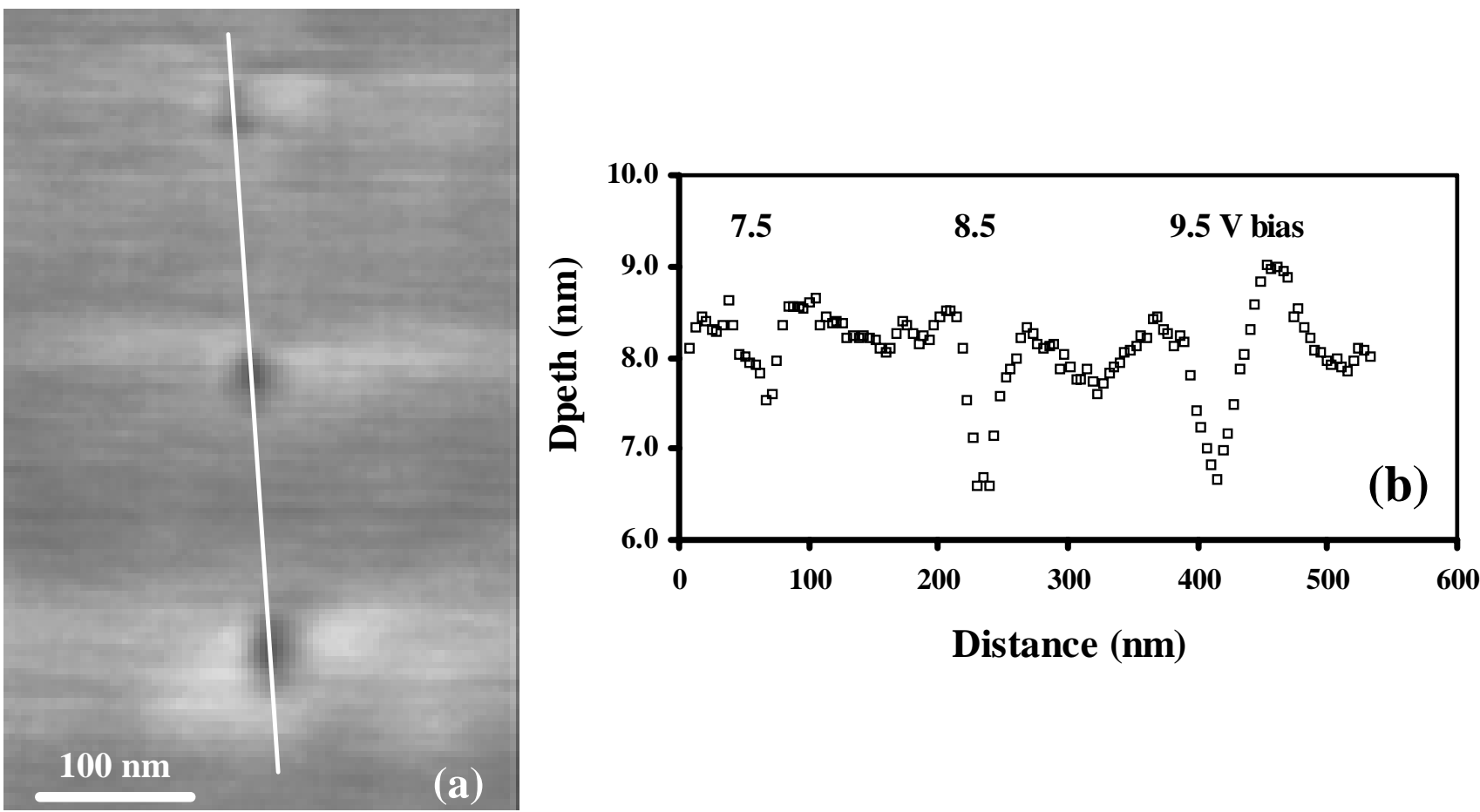

Figure 3 - Grey-scale image (top) and contour line (bottom) illustrating the dependence on energy deposition per unit area. The features were generated by applying a bias voltage to a stationary tip. 


\subsection{Tip-induced oxidation - Yield and effects on tip condition}

Tips with well-characterized initial conditions were used as probes for inducing local oxidation of Si and DLC. Yield, in terms of oxide growth, for $\mathrm{Si}$, and conversion of carbon to $\mathrm{CO}_{2}$, for DLC, was determined as a function of tip condition. As well, the effects on the tip of its use as an oxidative probe were investigated.

\subsubsection{Au-coated tip}

The tip was initially ohmic versus an Au substrate, while the $I-V$ data exhibited either direct or $\mathrm{F}-\mathrm{N}$ tunnelling, respectively, versus either DLC or Si+2.4 nm oxide surfaces. The outcome of a zero scan on DLC for $60 \mathrm{~s}$ at a sample bias of $5 \mathrm{~V}$ was a pit of ca. $300 \mathrm{~nm}$ width and $90 \mathrm{~nm}$ depth; the feature is essentially an indentation mirroring the shape of the tip, see contour line in figure 5 (b). The volume of converted material was therefore ca. $2 \times 106 \mathrm{~nm}^{3}$. The final state of the tip was subsequently checked by $I-V$ analysis against the Au-substrate, and found to be ohmic and identical to its initial state. The same tip was then used to generate local oxide growth on a Si wafer with a pre-existing $2.4 \mathrm{~nm}$ thermal oxide layer. A $6 \mathrm{~V}$ pulse was applied to a stationary tip, resulting in an oxide feature of ca. $100 \mathrm{~nm}$ FWHM and $10 \mathrm{~nm}$ height, figure 4 (a). The tip was also rastered over an area $1 \times 1 \mu \mathrm{m}^{2}$ at a sample bias of $6 \mathrm{~V}$, resulting in uniform oxide growth of height $10 \mathrm{~nm}$ over the rastered area, figure 5 (b). The oxide feature at the upper left corner of the raster is due to that being the default resting location of the tip.
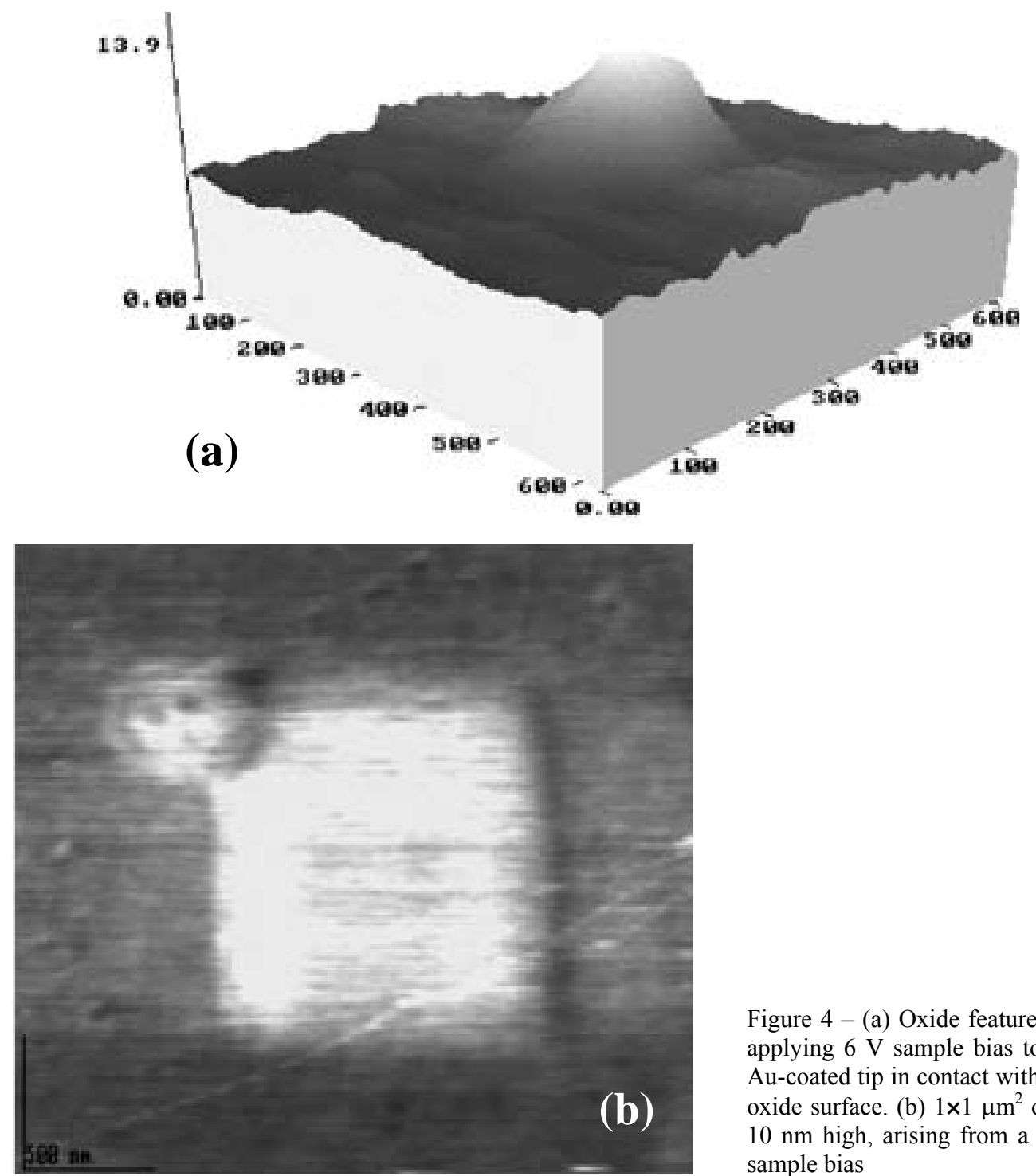

Figure 4 - (a) Oxide feature arising from applying $6 \mathrm{~V}$ sample bias to a stationary Au-coated tip in contact with a $\mathrm{Si}+2.4 \mathrm{~nm}$ oxide surface. (b) $1 \times 1 \mu \mathrm{m}^{2}$ oxide feature, $10 \mathrm{~nm}$ high, arising from a raster at $6 \mathrm{~V}$ sample bias 


\subsubsection{Si-tip with native oxide coating}

The tip was non-ohmic versus both $\mathrm{Au}$ and DLC substrates; the $I-V$ data reflected direct tunnelling through the native oxide layer. A zero scan was carried out on DLC, with identical conditions to those for the Au-coated tip. The yield was now considerably lower, producing a conical pit of ca. $150 \mathrm{~nm}$ width by $40 \mathrm{~nm}$ depth, equivalent to a volume of ca. $2 \times 10^{5} \mathrm{~nm}^{3}$, see image and contour line in figure 5 (a) and (b), respectively.
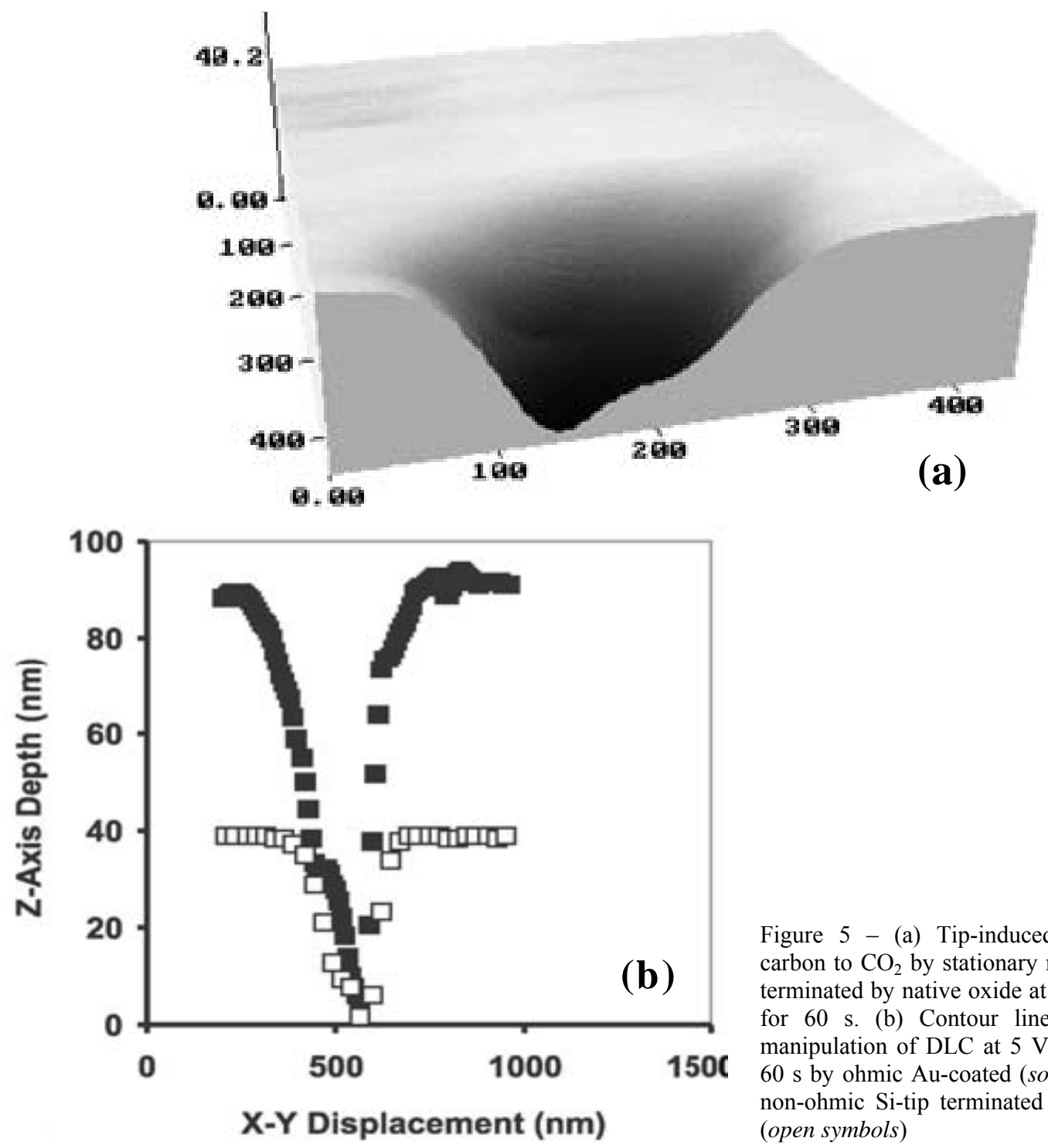

Figure 5 - (a) Tip-induced conversion of carbon to $\mathrm{CO}_{2}$ by stationary non-ohmic Si-tip terminated by native oxide at $5 \mathrm{~V}$ sample bias for $60 \mathrm{~s}$. (b) Contour lines of zero scan manipulation of DLC at $5 \mathrm{~V}$ sample bias for $60 \mathrm{~s}$ by ohmic Au-coated (solid symbols) and non-ohmic Si-tip terminated by native oxide (open symbols)

\subsection{DLC template and PDMS stamp formation}

As shown in the previous sections, by inducing thermal oxidation on a DLC surface, intricate details have been produced. The formation of features in the tens of $\mathrm{nm}$ have now been routinely reproduced. Figure 6 shows a lithographic pattern created by thermal oxidation. The DLC substrate is extremely durable, with the intricate details produced having a slope only limited by the aspect ratio of the tip $\left(<10^{\circ}\right.$ in this case). 


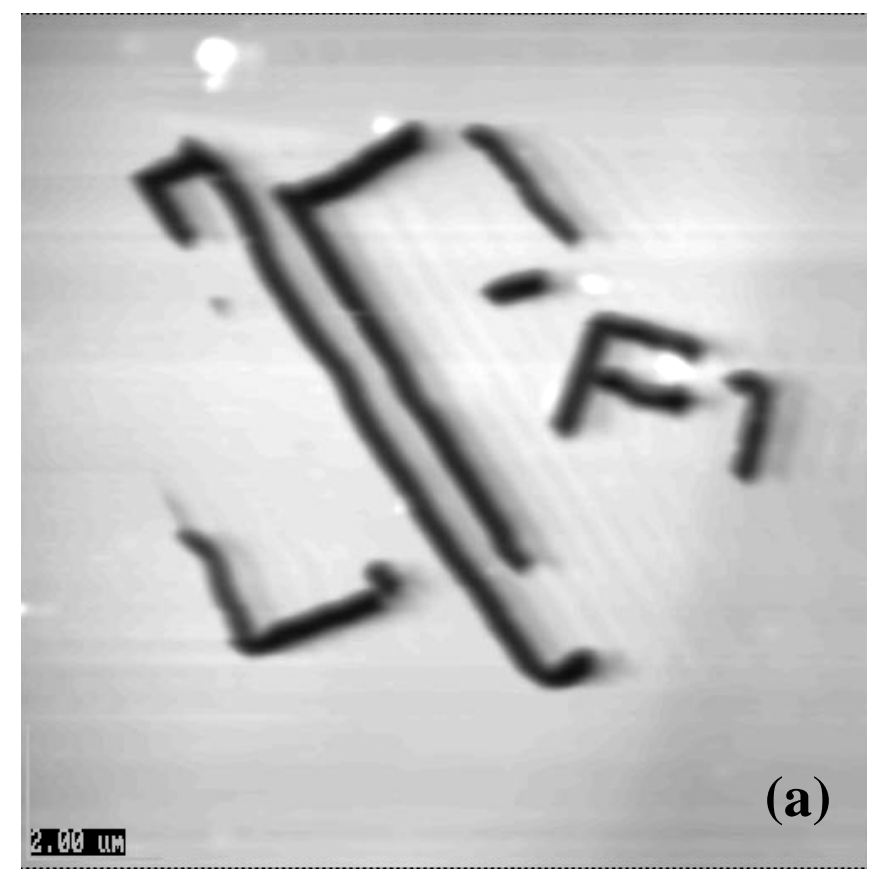

Figure 6 - DLC Template formed by tip-induced thermal oxidation.

The template created in figure 6 was coated with a layer of PDMS and thus used as a master. The PDMS was subsequently peeled off the DLC surface and imaged with a soft lever in order to avoid manipulation. The resultant fabricated PDMS stamp is shown in figure 7. The relief of the stamp correlates very well with the dimensions of the DLC master.

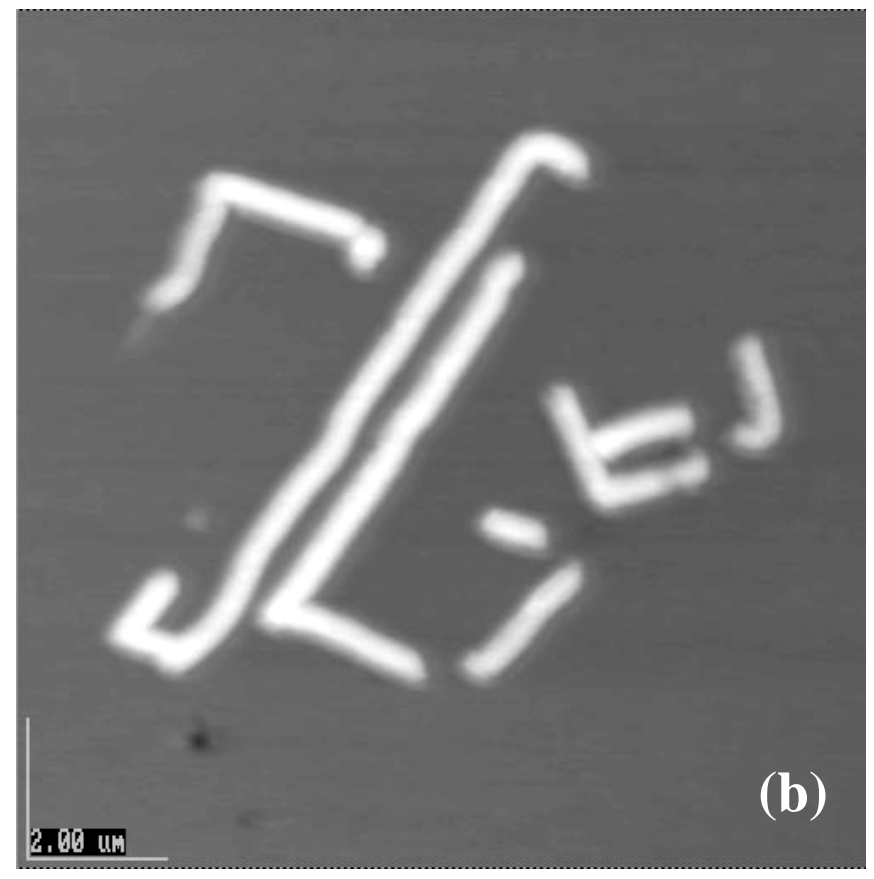

Figure 7 - The resultant PDMS structure -stamp formed by exposure of the polymer to the template topography. 
A 3 dimensional image of the PDMS stamp, and corresponding line profile along one of the longer stamp elements (A), are shown in figure 8 (a) and (b), respectively. The line profile perpendicular to one of the shorter elements (B), as shown in (a), for the PDMS stamp and the original DLC template are shown in figure 8 (c) and (d) The figure illustrates the $\mathrm{nm}$ scale surface roughness and height of the stamp (ca. $250 \mathrm{~nm}$ ) with (c) and (d) showing comparable dimensions of the master and resultant stamp.
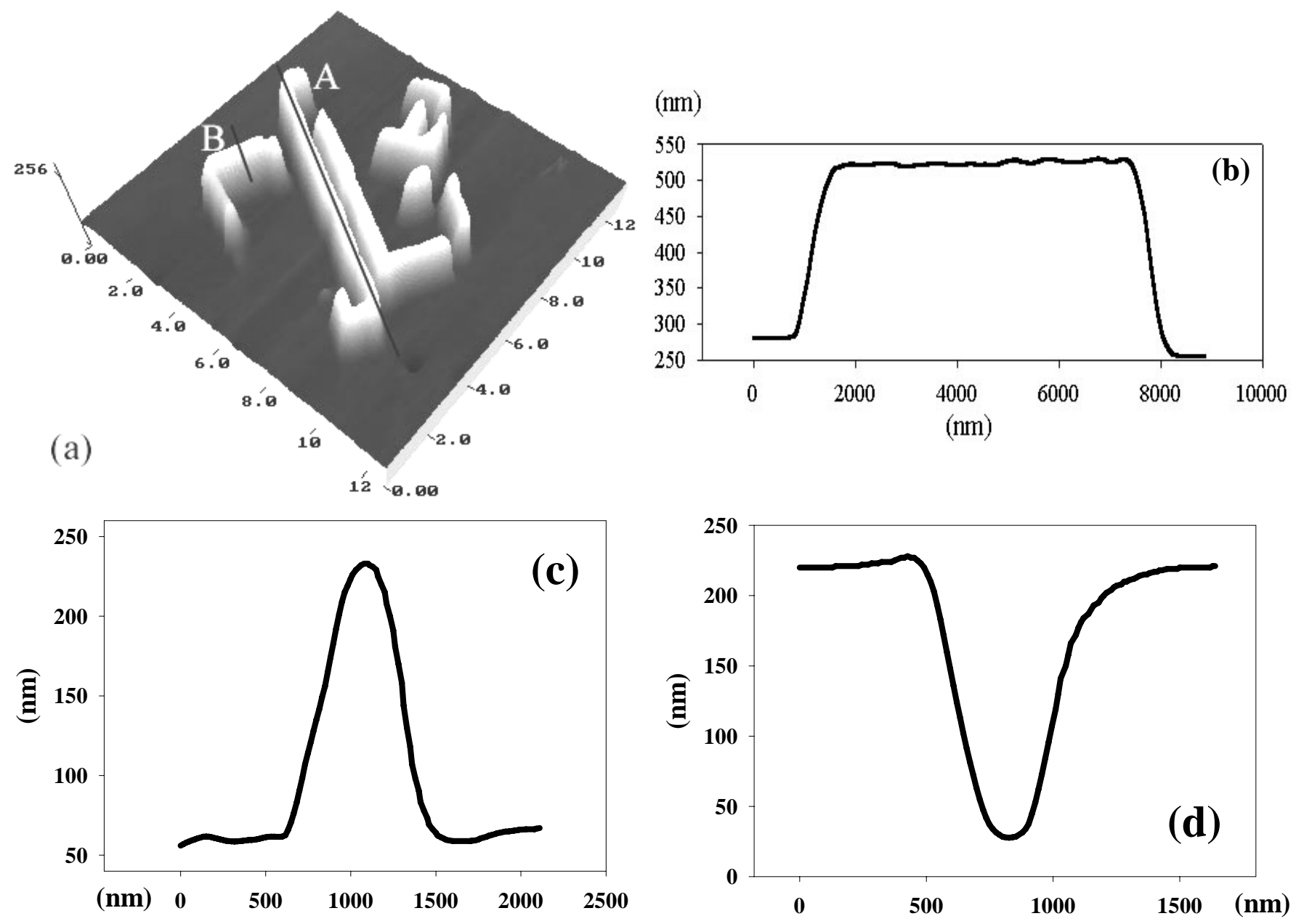

Figure 8 - (a) 3 dimensional representation of the stamp shown in figure 7 revealing more height detail. (b) shows a corresponding line profile showing the surface roughness of the stamp along one of the longer elements (A). (c) A line profile perpendicular to one of the shorter arms (B). (d) is a comparable line profile but taken on the original DLC master. The dimensions are comparable.

\section{CONCLUSION}

In this study we have demonstrated the use of Diamond-Like-Carbon (DLC) as a master template for producing polymer micro/nano stamps and 3-D polymer structures. Intricate surface relief patterns can be formed on the DLC surface from lithographic techniques by Atomic Force Microscopy (AFM) operated in the electrical conductivity mode.

Attributes of the technique as a template include

- Features with line widths less than $20 \mathrm{~nm}$ can be formed on the DLC.

- The radius of curvature at edges can be less than $10 \mathrm{~nm}$.

- The slope of the features can only be limited by the aspect ratio of the tip. 
- Highly complex shapes can be fashioned.

- $\quad$ Feature depth can be controlled by DLC film thickness and/or by the bias voltage applied.

- The master is highly durable.

- The master relief after patterning is extremely flat.

\section{ACKNOWLEDGEMENTS}

We would like to acknowledge Ray Sweatman for his assistance with spin-coating and with supplying the PDMS materials. The work was funded in part by a grant from the Australian Research Council.

\section{REFERENCES}

1. Dagata JA, Schneir J, Harary HH, Evans CJ, Postek MT, Bennett J, "Modification of hydrogen-passivated silicon by a scanning tunneling microscope operating in air", Applied Physics Letters, 56, 2001-2003, 1990.

2. Avouris P, Hertel T, Martel R, "Atomic force microscope tip-induced local oxidation of silicon: kinetics, mechanism, and nanofabrication”, Applied Physics Letters, 71, 285-287, 1997.

3. Myhra S, "Bias-induced spatially resolved growth and removal of Si-oxide by atomic force microscopy", Applied Physics A, 76, 63-69, 2003.

4. Bhushan B, "Micro/nanotribology using atomic force microscopy/Friction force microscopy : state of the art", Proc. Instn. Mech. Engrs J, 212, 1-18, 1998.

5. Vettiger P, Cross G, Despont M, Drechsler U, Durig U, Gotsmann B, Haberle W, Lantz MA, Rothuizen HE, Stutz R, Binnig GK, "The "millipede" - nanotechnology entering data storage", IEEE Transactions on Nanotechnology, 1, 39-55, 2002.

6. Kong L, Zhuang L, Chou S, "Writing and reading $7.5 \mathrm{Gbits} /$ in/ longitudinal quantized magnetic disk using magnetic force microscope tips", IEEE Transactions on Magnetics, 33, 3019-3021, 1997.

7. Shin H, Hong S, Moon J, Jeon JU, "Read/write mechanisms and data storage system using atomic force microscopy and MEMS technology", Ultramicroscopy, 91, 103-110, 2002.

8. Mühl T, Brückl H, Weise G, Reiss G, "Nanometer-scale lithography in thin carbon layers using electric field assisted scanning force microscopy", Journal of Applied Physics, 82, 5255-5258, 1997.

9. Mühl T, Brückl H, Kraut D, Kretz J, Mönch I, Reiss G, "Nanolithography of metal films using scanning force microscope patterned carbon masks", Journal of Vacuum Science and Technology B, 16, 3879-3882, 1998.

10. Mühl T, Kretz J, Mönch I, Schneider CM, "Parallel nanolithography in carbon layers with conductive imprint stamps", Applied Physics Letters, 76, 786-788, 2000.

11. Fang T-H, Weng C-I, Chiang MJ, "Effects of substrate bias on nanotribology of a-C:H films deposited by ECRMPCVD”, Diamond and Related Materials, 11, 1653-1659, 2002.

12. McDonald JC, Whitesides GM, "Poly(dimethylsiloxane) as a material for fabrication microfluidic devices", Accounts of Chemical Research, 35, 491-499, 2002.

13. Kane RS, Takayama S, Ostuni E, Ingber DE, Whitesides GM, "Patterning proteins and cells using soft lithography", Biomaterials, 20, 2363-2376, 1999.

14. Hu J, Deng T, Beck RG, Westervelt RM, Whitesides GM, "Fabrication of arrays of Schottky diodes using microtransfer molding", Sensors \& Actuators A, 75, 65-69, 1999.

15. Schueller OJA, Brittain ST, Whitesides GM, "Fabrication of glassy carbon microstructures by soft lithography", Sensors \& Actuators A, 72, 125-139, 1999.

16. Deng T, Prentiss M, Whitesides GM, "Fabrication of magnetic microfiltration systems using soft lithography", Applied Physics Letters, 80, 461-463, 2002.

17. Myhra S, Watson GS, "Tip-induced nano-writing/machining of Si and DLC surfaces-'anodic' versus thermal oxidation?", Applied Physics A, 81, 487-493, 2005.

18. McDonald JC, Whitesides GM, "Poly(dimethylsiloxane) as a material for fabricating microfluidic devices", Accounts of Chemical Research, 35, 491-499, 2002. 